\title{
Portuguese Validated Versions of the Alcohol Use Disorders Identification Test: A Systematic Review Protocol
}

\author{
Versões Validadas em Português do Alcohol Use Disorders \\ Identification Test: Protocolo de Revisão Sistemática da
}

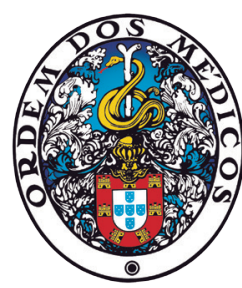
Literatura

\author{
Diogo Phalempin CARDOSO ${ }^{1}$, Daniela OLIVEIRA ${ }^{2}$, Beatriz ANTUNES ${ }^{3}$, Rosa SARAIVA ${ }^{4,5}$, Kathryn ANGUS ${ }^{6}$, \\ Eugenia GALLARDO ${ }^{5,7,8}$, Frederico ROSÁRIO $\triangle^{1,5,9}$ \\ Acta Med Port 2022 Apr;35(4):264-269 - https://doi.org/10.20344/amp.15765
}

\section{ABSTRACT}

Introduction: Alcohol consumption ranks among the top ten risk factors contributing to the global disease burden. Several international organizations recommend the use of the Alcohol Use Disorders Identification Test to screen for at-risk drinkers. However, a fully validated Portuguese version of this test is lacking. The aim of this study is to systematically review validated versions of the Alcohol Use Disorders Identification Test in the Portuguese language, the documented problems and solutions in its application and proposed cut-offs to identify at-risk drinkers.

Material and Methods: A systematic search will be performed in Ovid MEDLINE, CINAHL, PsycINFO, ÍndexRMP, LILACS, African Journals Online and SciELO databases, along with grey literature searches to identify validation studies of the AUDIT in Portuguese. Two authors will independently extract data and assess the studies' methodological quality, using QUADAS-2 and CASP checklists. Conclusion: This review will provide important information on the validity of the Alcohol Use Disorders Identification Test as a screening tool for at-risk drinking in Portugal and other official Portuguese speaking countries.

Keywords: Alcoholism/epidemiology; Alcohol-Induced Disorders; Alcohol-Related Disorders; Mass Screening

\section{RESUMO}

Introdução: O consumo de álcool é um importante fator de risco modificável. Várias organizações internacionais recomendam a utilização do Alcohol Use Disorders Identification Test para identificar consumidores excessivos de álcool. No entanto, não parece haver uma versão totalmente validada deste questionário em português. O objetivo deste estudo é identificar versões validadas do Alcohol Use Disorders Identification Test em português, problemas e soluções na sua aplicação, e pontos de corte para identificar consumidores excessivos.

Material e Métodos: Será realizada uma revisão sistemática dos estudos de validação do AUDIT em português existentes nas bases de dados Ovid MEDLINE, CINAHL, PsycINFO, ÍndexRMP, LILACS, African Journals Online e SciELO, bem como na literatura cinzenta. Dois autores extrairão informação, e avaliarão a qualidade dos estudos selecionados, de forma independente, utilizando as grelhas QUADAS-2 e CASP.

Conclusão: Esta revisão irá fornecer informação relevante sobre a validade do Alcohol Use Disorders Identification Test como método de rastreio do consumo excessivo de álcool em Portugal e noutros países de língua oficial portuguesa.

Palavras-chave: Alcoolismo/epidemiologia; Perturbações Induzidas por Álcool; Perturbações Relacionados ao Uso de Álcool; Rastreio

\section{INTRODUCTION}

Alcohol use is one of the top ten risk factors for disease and disability. It is estimated that $5.3 \%$ of all deaths and $5.1 \%$ of all disability-adjusted life years (DALYs) are attributable to alcohol use, surpassing other leading causes of death, such as HIVIAIDS, tuberculosis and diabetes. ${ }^{1}$ The effect on premature mortality is even higher, accounting for $7.2 \%$ of all deaths, with a disproportionate effect on younger populations $-13.5 \%$ of all deaths between 20 - 39 years of age $^{1}$ - with considerable variability between countries. Portugal ranks $13^{\text {th }}$ in the World Health Organization's (WHO)

list of countries with the highest total alcohol per capita consumption. ${ }^{1}$ According to the Portuguese DirectorateGeneral of Health, alcohol was the third leading cause of premature death in 2018 and was responsible for $6.4 \%$ of all premature deaths and $8.7 \%$ of all DALYs. ${ }^{2}$ Efforts must be undertaken to lower the overall population level of alcohol consumption.

Screening and brief interventions for alcohol at the level of primary health care are effective and cost-effective in reducing alcohol-related harm. ${ }^{3-7}$ The $\mathrm{WHO}^{8}$ and several

1. Unidade de Saúde Familiar Tondela. Agrupamento de Centros de Saúde Dão Lafões. Viseu. Portugal.

2. Unidade de Saúde Familiar Grão Vasco. Agrupamento de Centros de Saúde Dão Lafões. Viseu. Portugal.

3. Unidade de Saúde Familiar Vouzela. Agrupamento de Centros de Saúde Dão Lafões. Viseu. Portugal.

4. Serviço de Epidemiologia e Saúde Pública. Centro Hospitalar Universitário Cova da Beira. Covilhã. Portugal.

5. Comissão Executiva para a Área de Missão Problemas Ligados ao Álcool. Centro Académico e Clínico das Beiras. Covilhã. Portugal.

6. Institute for Social Marketing \& Health. Faculty of Health Sciences \& Sport. University of Stirling. Stirling. Scotland. United Kingdom.

7. Centro de Investigação em Ciências da Saúde. Faculdade de Ciências da Saúde. Universidade da Beira Interior. Covilhã. Portugal.

8. Laboratório de Fármaco-Toxicologia. Universidade da Beira Interior. Covilhã. Portugal.

9. Equipa de Projeto em Investigação. Agrupamento de Centros de Saúde Dão Lafões. Viseu. Portugal.

$\triangle$ Autor correspondente: Frederico Rosário. fredmbr@gmail.com

Recebido/Received: 20/01/2021 - Aceite/Accepted: 18/05/2021 - Publicado Online/Published Online: 18/08/2021 - Publicado/Published: 01/04/2022 Copyright @ Ordem dos Médicos 2022 
national and international guidelines ${ }^{9-11}$ recommend the use of the Alcohol Use Disorders Identification Test (AUDIT) to identify at-risk drinkers. The AUDIT was developed in the 1980 s as a means to address an unmet need: to have a simple instrument that could be used by healthcare workers in both developing and developed countries to identify hazardous and harmful drinkers. ${ }^{12}$ Since its publication, the AUDIT has been extensively researched concerning its psychometric properties and performance characteristics and became one of the most widely used tools for assessing risky drinking. ${ }^{13,14}$ Research shows that both the AUDIT-C (its abridged version, containing only the first three questions $)^{15}$ and the full 10 -item questionnaire can be successfully used to screen for alcohol use disorders. ${ }^{16}$

Notwithstanding its usefulness as a screening tool, researchers worldwide have pointed out some limitations to the AUDIT's validity and accuracy. Among these limitations are concerns regarding the validity of the individual items across different cultural and demographic groups and different languages, the possible differences regarding concepts such as 'standard drink', 'typical day' or 'heavy drinking session' in these groups, and the optimal cut-off for determining at-risk drinking. ${ }^{13}$ Several studies focusing on the validity and performance characteristics of the AUDIT for different populations have been published, showing acceptable validity and reliability across a wide range of settings. ${ }^{16-21}$ To our knowledge, only one such study was conducted in Portugal, in which Roque da Cunha used translation and backtranslation to obtain a valid Portuguese language version of the AUDIT questionnaire. ${ }^{23}$ Based on this study, the AUDIT was included in the Portuguese national guidelines for 'Early Detection and Brief Intervention in Alcohol Consumption' as the recommended tool to screening for at-risk drinkers. ${ }^{17}$ However, there are a number of limitations to the current version of the Portuguese AUDIT, such as being based on a small, non-representative sample of under 65 year-old subjects, or not having determined the best cut-off points. ${ }^{18}$ This could have several implications in practice. For instance, not determining the best cut-off points may increase the number of low-risk drinkers classified as high-risk drinkers (false positives); conversely, and potentially more problematic, it may also increase the number of high-risk drinkers that are classified as low-risk drinkers (false negatives) and to whom counselling is not, therefore, offered. A number of systematic reviews focused on the AUDIT's validity have been published, ${ }^{19-23}$ but none included Portuguese data that could answer the above-mentioned limitations. ${ }^{24} \mathrm{~A}$ systematic review focused on Portuguese versions of the AUDIT is, therefore, needed to strengthen the recommendation to use the AUDIT as a screening tool for at-risk drinkers in Portugal, which could contribute to its more widespread use in clinical practice. The present review is part of the MARADONA (Making Advances to Recognize Alcohol use and alcohol-related disorders by Developing Old and New Assessment tools) research project, which aims to improve the way by which people with excessive alcohol consumption and alcohol-related disorders are identified by develop- ing new, or validating existing, screening tools.

\section{Objectives}

This study aims to systematically review Portuguese versions of the AUDIT questionnaire, in order to identify validated versions of the test, the documented problems and solutions in its application and proposed cut-offs to identify at-risk drinkers. The specific research questions we will address are as follows:

1. What Portuguese versions of the AUDIT exist globally and what are the differences between them?

2. What are the documented problems and solutions in the application of the AUDIT in Portuguese-speaking countries?

3. What validation studies of Portuguese versions of the AUDIT exist?

4. What are the proposed cut-offs of Portuguese versions of the AUDIT for screening for at-risk drinkers?

\section{MATERIAL AND METHODS}

The review methods outlined here are in accordance with the Preferred Reporting Items for Systematic Review and Meta-Analysis Protocols (PRISMA-P) statement [see Appendix file 1 for completed PRISMA-P checklist (Appendix 1: https://www.actamedicaportuguesa.com/revista/ index.php/amp/article/view/15765/Appendix_01.pdf)]. ${ }^{25,26}$ The study protocol was pre-registered on PROSPERO (registration number CRD42021223631).

\section{Inclusion and exclusion criteria}

Study design: Quantitative and qualitative studies reporting primary data will be eligible for inclusion. Editorials, opinion pieces and letters to the editor will be excluded.

Participants: No limitations regarding the participants will be applied.

Outcomes: The outcomes of interest in this review are Portuguese versions of the AUDIT that use a translationback translation methodology with or without assessments of its psychometric properties (e.g. content and construct validity, internal consistency) and/or performance characteristics (e.g. accuracy, sensitivity, specificity) at the optimal cut-off (please see Tables 1 and 2 for definitions of the psychometric properties and performance characteristics to be retrieved). Where possible, we will also retrieve information on the problems faced, and solutions found, during its application. Studies will be excluded if: the AUDIT is used or referred to, but the study's purpose was not its validation; if it uses a methodology other than translation-back translation; and if the study was conducted in countries other than those mentioned below.

Setting: No limitations regarding the type of setting shall be applied provided the study was performed in countries with Portuguese as an official spoken language (Angola, Brazil, Cape Verde, East Timor, Equatorial Guinea, GuineaBissau, Mozambique, Portugal and São Tomé and Príncipe). Studies conducted in other countries will be excluded.

Language: We will include articles published in 
Spanish, English and Portuguese. Potentially relevant studies published in other languages will be excluded from the analysis but their titles will be listed in a table.

\section{Information sources and search strategy}

The following electronic databases will be searched, from 1987 (the year when the first WHO report on the AUDIT was made available) ${ }^{12}$ until December 2020 , for studies meeting the inclusion criteria stated above: Ovid MEDLINE, CINAHL, PsycINFO, ÍndexRMP, LILACS, African Journals Online and SciELO. We will also perform a grey literature search through the following: 1) grey literature databases, by searching the OpenDOAR database (global Directory of Open Access Repositories) for repositories of countries relevant for this study (at the time of writing, this database included 206 repositories of relevance to the review); 2) Google Scholar; and 3) consultation with experts, which includes authors of relevant conference abstracts. When available, the abstract of all eligible articles will be used to perform a search using the Journal/Author Name Estimator (JANE) search engine to access similar, possibly relevant, articles. To ensure literature saturation, we will scan the reference lists of relevant systematic reviews and metaanalyses identified through the search for potentially eligible papers. The search strategy will be developed together with two health information specialists (RS and KA), based on a list of relevant keywords identified from an exploratory search of the literature and by exploring the Medical Subject Headings (MeSH terms) of the US National Library of Medicine. The final search will be performed by KA and RS after adapting the Ovid MEDLINE strategy to the syntax of the other databases [see Appendix 2 for the complete Ovid MEDLINE search strategy (Appendix 2: https://www. actamedicaportuguesa.com/revista/index.php/amp/article/ view/157658/Appendix_02.pdf)].

Table 1 - Definition of the psychometric properties to be extracted

\begin{tabular}{|c|c|c|}
\hline Psychometric Domain & Psychometric property & Definition \\
\hline \multirow{4}{*}{$\begin{array}{l}\text { Reliability: the degree to which } \\
\text { the measurement is free from } \\
\text { measurement error }\end{array}$} & Parallel forms reliability & $\begin{array}{l}\text { Obtaining the same results by administering different versions of } \\
\text { an assessment tool to the same group of individuals. }\end{array}$ \\
\hline & Internal consistency & The degree of interrelatedness among the items. \\
\hline & Inter-rater reliability & $\begin{array}{l}\text { The degree of consistency between different people conducting } \\
\text { the same test. }\end{array}$ \\
\hline & Test-retest reliability & The consistency of the same test applied over time. \\
\hline \multirow{4}{*}{$\begin{array}{l}\text { Validity: the degree to which an } \\
\text { instrument measures the constructs } \\
\text { it intends to measure }\end{array}$} & Content validity & $\begin{array}{l}\text { The degree to which the content of an instrument is an adequate } \\
\text { reflection of the construct to be measured. }\end{array}$ \\
\hline & Construct validity & $\begin{array}{l}\text { The degree to which the test measures the particular construct } \\
\text { that it is designed to measure. }\end{array}$ \\
\hline & Face validity & $\begin{array}{l}\text { The degree to which an instrument indeed appears to be an } \\
\text { adequate reflection of the construct to be measured. }\end{array}$ \\
\hline & Criterion validity & $\begin{array}{l}\text { The degree to which the scores of an instrument are an adequate } \\
\text { reflection of a "gold standard". }\end{array}$ \\
\hline
\end{tabular}

Table 2 - Definition of the performance characteristics to be extracted

\begin{tabular}{|c|c|c|}
\hline Performance Domain & Performance measures & Definition \\
\hline \multirow{8}{*}{$\begin{array}{l}\text { Accuracy: the ability of a test } \\
\text { to detect a condition when it is } \\
\text { present and detect the absence } \\
\text { of a condition when it is absent }\end{array}$} & Sensitivity & $\begin{array}{l}\text { The frequency with which a test correctly generates a positive result } \\
\text { in a situation where the condition being tested exists. }\end{array}$ \\
\hline & Specificity & $\begin{array}{l}\text { The frequency with which a test correctly generates a negative } \\
\text { result in a situation where the condition being tested does not exist. }\end{array}$ \\
\hline & Positive predictive value & $\begin{array}{l}\text { The chance that a condition being tested for actually exists when } \\
\text { tested positive for. }\end{array}$ \\
\hline & Negative predictive value & $\begin{array}{l}\text { The chance that a condition being tested for does not actually exist } \\
\text { when tested negative for. }\end{array}$ \\
\hline & Positive likelihood ratio & $\begin{array}{l}\text { The probability of a person who has the condition testing positive } \\
\text { (sensitivity) divided by the probability of a person who does not } \\
\text { have the condition testing positive ( } 1 \text { - specificity). }\end{array}$ \\
\hline & Negative likelihood ratio & $\begin{array}{l}\text { The probability of a person who has the condition testing negative } \\
(1-\text { sensitivity) divided by the probability of a person who does not } \\
\text { have the condition testing negative (specificity). }\end{array}$ \\
\hline & Area under the ROC curve & $\begin{array}{l}\text { A performance measurement for the classification problems at } \\
\text { various threshold settings. ROC is a probability curve and AUC } \\
\text { represents the degree or measure of separability. }\end{array}$ \\
\hline & Diagnostic odds ratio & $\begin{array}{l}\text { The ratio of the odds of positivity in subjects with the condition } \\
\text { relative to the odds in subjects without the condition. }\end{array}$ \\
\hline
\end{tabular}




\section{Data management and study selection}

The results of the literature search will be uploaded to the EndNote X9 software. One reviewer (DPC) will scan the titles and/or abstracts to eliminate duplicate results. Afterwards, two reviewers will independently screen titles and abstracts of identified references: half of the studies by DPC and DO, the other half by DPC and BA. Studies will be excluded if they: 1) Do not have a title; 2) A full-text copy cannot be obtained; 3) Are not published in one of the following languages: English, Spanish or Portuguese; 4) Are not aimed at validating the AUDIT questionnaire; 5) Were conducted in countries where Portuguese is not an official language. Disagreements will be resolved through consensus. If consensus cannot be reached, a third reviewer (FR) will be contacted. Full-text copies of all studies meeting inclusion criteria and of those which eligibility is unclear after assessment of title and abstract will be sought and the selection process repeated. If any of the full-text articles cannot be retrieved, a list detailing the unobtainable articles will be provided. Reasons for excluding papers from the analysis will be recorded in a table describing the characteristics of the studies excluded. Reviewers will not be blinded for any aspect of the studies identified and selected.

\section{Data extraction}

Two reviewers, using the same above-mentioned distribution methodology, will independently extract data to a data extraction form specifically designed for this review and later entered into a Microsoft Excel spreadsheet. Disagreements will be resolved as described above. Data to be extracted will include: first author; year of publication; title; language of publication; country of origin; primary objective of the study; characteristics of the study sample (such as age and sex distribution); definition of standard drink; definition of a single occasion of drinking; examples of standard drink and results of validation (which may include any or all of the following: (i) qualitative analysis on the perception of the AUDIT questions (interviews with experts and/or patients); (ii) reference to a predetermined protocol of systematic translation and back-translation of the tool; (iii) pilot study on the feasibility of the AUDIT; (iv) assessment of the dimensions of the AUDIT; (v) psychometric properties (reliability, validity and responsiveness); and (vi) performance characteristics (optimal cut-off point, accuracy, sensitivity, specificity, ROC curve analysis), separating results by sex, age group and any other variables of interest as reported in the retrieved studies.

\section{Assessment of methodological quality}

A critical appraisal of the validity of the included studies will be conducted to assist our synthesis of the evidence. Two reviewers will independently assess the methodological quality of the studies selected for this systematic review, using the same above-mentioned distribution methodology. Disagreements will be resolved as described above. The quality of quantitative studies will be appraised with the Quality Assessment of Diagnostic Accuracy Studies (QUADAS-2) tool. ${ }^{27}$ Qualitative studies will be assessed with the Critical Appraisal Skills Programme (CASP) qualitative research checklist. ${ }^{28}$

Primary search in Medline, CINHAL, PsycINFO, LILACS, AJOL, Scielo, Grey Literature and JANE results in ___ potentially relevant references

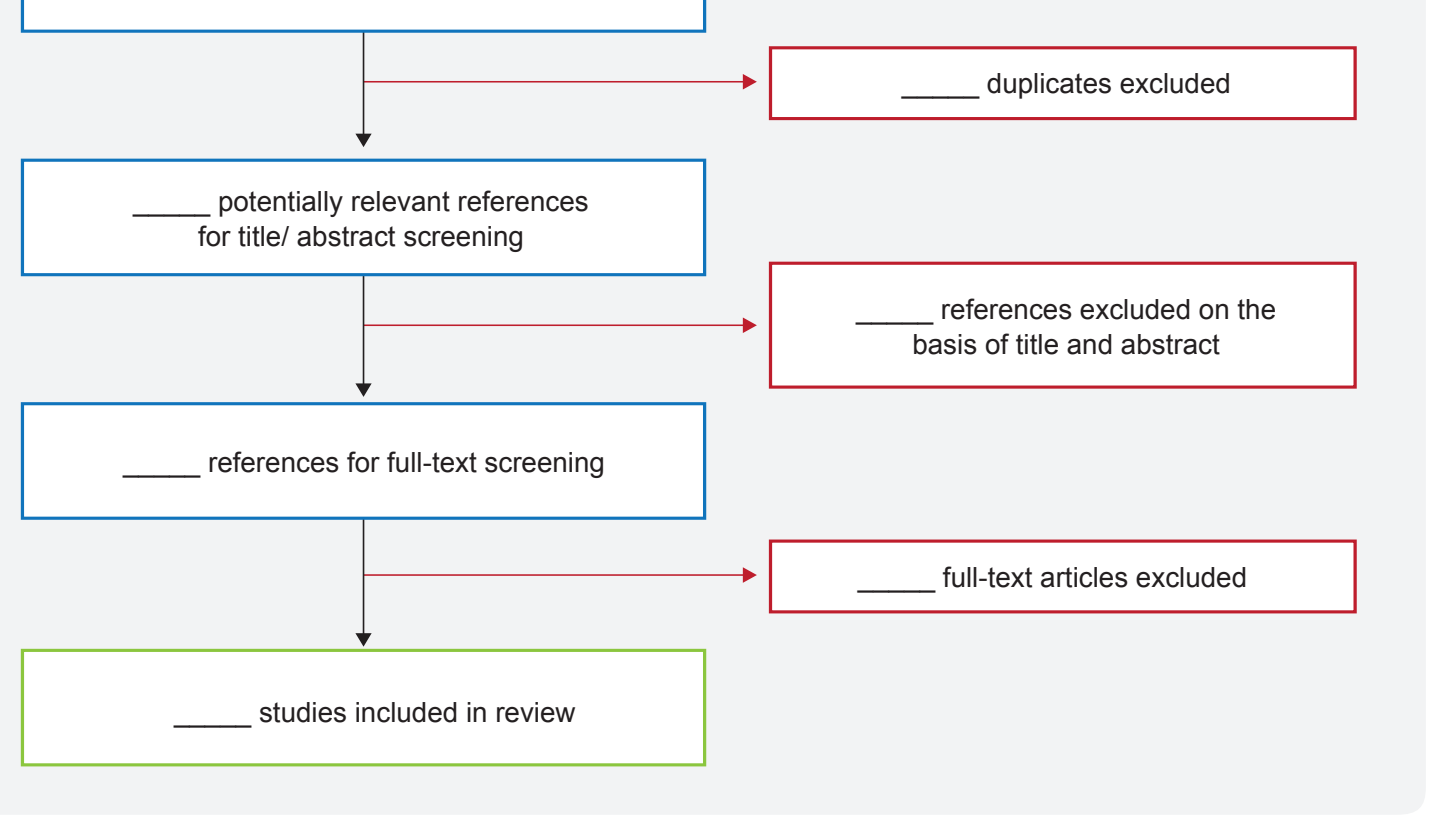

Figure 1 - Flow diagram of screening process 


\section{Data synthesis}

This review will be reported in accordance with the PRISMA-DTA guidelines which will include a flow diagram (Fig. 1) and a table detailing the studies selected. The review will start by reporting the results of the literature searched. PRISMA flowcharts and tables will be presented with reasons for inclusion and exclusion as well as the methodology of studies included. The results of the review will be reported in a table and a narrative synthesis of the findings will be provided. Due to the expected heterogeneity of the populations in the included studies, we do not intend to perform a meta-analysis of the collected data.

\section{DISCUSSION}

This review will aggregate validated versions of the AUDIT in Portuguese, highlighting obtained cut-off values for different levels of consumption, problems associated with its application and any relevant questions added to its base format. The results of this review could be used for a scientifically-sound and thorough update of the Portuguese guidelines on alcohol screening and brief intervention, which in turn could prove useful to support the implementation of alcohol screening in daily practice in Portugal. Several barriers have been identified regarding the implementation of alcohol screening tools, which could be directly related to the AUDIT (such as unfamiliarity with the questionnaire or lack of trust in its reliability) or indirectly related to it (such as believing patients will not follow the advice to cut down or fear of damaging the therapeutic relationship with the patient). ${ }^{29,30}$ While it is not within the scope of this review to identify the underlying causes of the resistance of healthcare professionals to using the AUDIT, we believe that the results from this review could contribute to addressing these barriers.

Possible limitations to this review include the exclusion of articles published in languages other than English,
Portuguese and Spanish, and of validation studies in countries in which the official spoken languages do not include Portuguese. Notwithstanding, we do not anticipate that many studies, if any, will be excluded due to the abovementioned reasons: firstly, the vast majority of Portugueseled research is published in these three languages; and secondly, it is unlikely that validation studies on the AUDIT have been conducted in countries in which Portuguese is not the official language. Finally, another limitation is that, since there is no established methodology for researching the grey literature, it is possible that some relevant unpublished papers will not be captured with our search strategy.

\section{CONCLUSION}

To our knowledge, this will be the first study to review Portuguese versions of the AUDIT questionnaire, which could be used to support clinicians and decision-makers in their efforts to implement the AUDIT as a screening tool for at-risk drinking in Portugal and other official Portuguese speaking countries.

\section{AUTHORS CONTRIBUTION}

DPC: Conception and design of the paper and the research strategy. Draft of the article.

DO, BA: Critical review of the article.

RS, KA: Conception of the research strategy, critical review of the article.

EG: Critical review of the article.

FR: Conception of the study and the research strategy. Protocol design. Draft of the article.

\section{COMPETING INTERESTS}

None.

\section{FUNDING SOURCES}

This study has no funding.

\section{REFERENCES}

1. World Health Organization. Global status report on alcohol and health 2018. Report No.: CC BY-NC-SA 3.0 IGO. Geneva: WHO; 2018.

2. Direção-Geral da Saúde. A Saúde dos Portugueses. Lisboa: DGS; 2016.

3. Angus C, Thomas C, Anderson P, Meier P, Brennan A. Estimating the cost-effectiveness of brief interventions for heavy drinking in primary health care across Europe. Eur J Public Health. 2017;27:345-51.

4. Solberg L, Maciosek M, Edwards N. Primary care intervention to reduce alcohol misuse ranking its health impact and cost effectiveness. Am J Prev Med. 2008;34:143-52.

5. Angus $\mathrm{C}$, Latimer $\mathrm{N}$, Preston L, Li J, Purshouse R. What are the implications for policy makers? A systematic review of the costeffectiveness of screening and brief interventions for alcohol misuse in primary care. Front Psychiatry. 2014;5:114.

6. Angus C, Scafato E, Ghirini S, Torbica A, Ferre F, Struzzo P, et al. Costeffectiveness of a programme of screening and brief interventions for alcohol in primary care in Italy. BMC Fam Pract. 2014;15:26.

7. Wutzke S, Shiell A, Gomel M, Conigrave K. Cost effectiveness of brief interventions for reducing alcohol consumption. Soc Sci Med. 2001;52:863-70.

8. Babor T, Higgins-Biddle J, Saunders J. The alcohol use disorders identification test: guidelines for use in primary care. Geneva: WHO 2001.

9. Anderson P, Gual A, Colom J. Alcohol and primary health care:

clinical guidelines on identification and brief interventions. Barcelona: Department of Health of the Government of Catalonia; 2005.

10. O'Connor E, Perdue L, Senger C, Rushkin M, Patnode C, Bean S, et al. Screening and behavioral counseling interventions to reduce unhealthy alcohol use in adolescents and adults: updated evidence report and systematic review for the US Preventive Services Task Force. JAMA. 2018;320:1910-28.

11. Chang J, Kim J, Gyu Jung J, Kim S, Yoon S, Jang H. Validity of alcohol use disorder identification test-Korean revised version for screening alcohol use disorder according to Diagnostic and Statistical Manual of Mental Disorders, Fifth Edition Criteria. Korean J Fam Med. 2016;37:323-8

12. Saunders $\mathrm{J}$, Aasland $\mathrm{O}$. WHO Collaborative Project on the Identification and Treatment of Persons with Harmful Alcohol Consumption. Geneva: World Health Organization; 1987.

13. Reinert D, Allen J. The alcohol use disorders identification test: an update of research findings. Alcohol Clin Exp Res. 2007;31:185-99.

14. World Health Organization. WHO Collaborative Project on Identification and Management of Alcohol-Related Problems in Primary Health Care: report on phase IV: development of country-wide strategies for implementing early identification and brief intervention in primary health care. Geneva: WHO; 2006.

15. Saunders J, Aasland O, Amundsen A, Grant M. Alcohol consumption and related problems among primary health care patients: WHO 
collaborative project on early detection of persons with harmful alcohol consumption-I. Addiction. 1993;88:349-62.

16. Dawson D, Smith S, Saha T, Rubinsky A, Grant B. Comparative performance of the AUDIT-C in screening for DSM-IV and DSM-5 alcohol use disorders. Drug Alcohol Depend. 2012;126:384-8.

17. Direção-Geral da Saúde. Norma $n^{\circ} 30 / 2012$ de 28 de Dezembro, Atualização 18/12/2014. Lisboa. [accessed 2021 Mar 17]. Available from: https://www.dgs.pt/normas-clinicas/normas-clinicas.aspx.

18. Roque da Cunha J. Validação da versão portuguesa dos questionários AUDIT e Five-shot para identificação de consumo excessivo de álcool. Lisboa: Internato Complementar de Clínica Geral da Zona Sul; 2002.

19. Bohn M, Babor T, Kranzler H. The Alcohol Use Disorders Identification Test (AUDIT): validation of a screening instrument for use in medical settings. J Stud Alcohol. 1995;56:423-32.

20. Garcia Carretero M, Novalbos Ruiz J, Martinez Delgado J, O'Ferrall Gonzalez C. Validation of the Alcohol Use Disorders Identification Test in university students: AUDIT and AUDIT-C. Adicciones. 2016;28:194204.

21. Hallit J, Salameh $P$, Haddad C, Sacre $H$, Soufia M, Akel M, et al. Validation of the AUDIT scale and factors associated with alcohol use disorder in adolescents: results of a National Lebanese Study. BMC Pediatr. 2020;20:205.

22. Khadjesari Z, White IR, McCambridge J, Marston L, Wallace P, Godfrey $C$, et al. Validation of the AUDIT-C in adults seeking help with their drinking online. Addict Sci Clin Pract. 2017:12:2.

23. Levola J, Aalto M. Screening for at-risk drinking in a population reporting symptoms of depression: a validation of the AUDIT, AUDIT-C, and AUDIT-3. Alcohol Clin Exp Res. 2015;39:1186-92.

24. Ferreira J, Martins J, Coelho M, Kahler C. Validation of Brief Young Adult Alcohol Consequences Questionnaire (B-YAACQ): Portuguese version. Span J Psychol. 2014;17:E71.

25. Moher D, Shamseer L, Clarke M, Ghersi D, Liberati A, Petticrew M, et al. Preferred reporting items for systematic review and meta-analysis protocols (PRISMA-P) 2015 statement. Syst Rev. 2015;4:1.

26. Shamseer L, Moher D, Clarke M, Ghersi D, Liberati A, Petticrew M, et al. Preferred reporting items for systematic review and meta-analysis protocols (PRISMA-P) 2015: elaboration and explanation. BMJ. 2015;350:g7647.

27. QUADAS-2: 2010. [accessed 2020 Sep 22]. Available from: https://www. bristol.ac.uk/population-health-sciences/projects/quadas/quadas-2/.

28. CASP Checklists: 2017. [accessed 2020 Sep 22]. Available from: https:// casp-uk.net/casp-tools-checklists/.

29. Johnson M, Jackson R, Guillaume L, Meier P, Goyder E. Barriers and facilitators to implementing screening and brief intervention for alcoho misuse: a systematic review of qualitative evidence. J Public Health 2011;33:412-21.

30. Rosario F, Santos M, Angus K, Pas L, Ribeiro C, Fitzgerald N. Factors influencing the implementation of screening and brief interventions for alcohol use in primary care practices: a systematic review using the COM-B system and Theoretical Domains Framework. Implement Sci. 2021;16:6 